\title{
E-COMMERCE SECURITY: NO SILVER BULLET
}

Anup K. Ghosh

\subsection{INTRODUCTION}

Electronic commerce has come out of its infancy, re-buffed its nay-sayers, and is now a multi-billion dollar industry. The success of early adopters in electronic commerce has now led the pragmatist herd in participating in on-line commerce. It is now a staple of advertising to include a World Wide Web address for consumers to learn more about a company or product and even to perform on-line transactions.

With the convergence of businesses to the Web and with the growth of on-line commerce, new threats to corporate assets have emerged through vulnerabilities in computer security. It is important to note that in the physical world, security and trust in commerce have evolved over centuries through a system of expectations, contracts, and a judicial system for enforcement. When depositing money with a bank, traditionally people expected that their money is adequately protected in bank vaults. In reality, most money is stored on electronic ledgers with wire transfers used for payment settlement between parties. However, the perception of security together with depositors' insurance provides trust in the banking system for consumers.

On the Internet, new forms of currency are evolving to support electronic payment. Some payment systems involve supporting the existing infrastructure for credit and debit transactions, while other evolving payment systems provide payment by means of digital currency [3]. Whether the solution is new cryptographic protocols for assuring the integrity of payment, or simply building an Internet interface to existing payment infrastructures, trust in Internet-based 
payment systems is required to build the consumer base for electronic commerce.

In physical world transactions, rarely do we ask for secure phone lines for placing orders, rarely must we provide proof-positive identification when giving out credit card numbers, rarely are non-repudiation systems employed, and rarely do we worry about our credit card slips we give to waiters in restaurants. It is reasonable therefore to ask why we must go to extraordinary lengths to secure e-commerce systems. One simple reason is perception. Security violations in Internet-based systems have received much notoriety in the popular press which, in turn, feeds the media frenzy over every new Internet security violation. As a result, a general paranoia of insecurity in e-commerce transactions has gripped the consumer public. Aside from perception, there are several technical reasons why electronic commerce must have stronger requirements on security than traditional forms of commerce [8]. First, and most importantly, is the inter-networking of computer systems. This topic is discussed shortly in this introduction. Second, the storage of sensitive data in repositories or databases makes e-commerce systems ideal targets. For instance, hacking an on-line firm's database that holds all its customers' credit card numbers is more profitable than dumpster diving for credit card receipts. Third, the lack of forensic evidence in computer crimes makes detection, capture, and prosecution more difficult. Good and regular auditing of computer usage is rarely practiced. Legal cases against computer crimes depend on auditing practices, audit trails, and the ability to demonstrate malice. Fourth, the ability to write programs to automate computer crimes provides a higher return on investment for computer criminals than physically committing the crime on site. Once written, hacking tools are distributed widely among "underground" networks and used by junior hackers that often do not know how the exploit scripts work, let alone how to write them. Finally, computer crimes can be committed thousands of miles from the crime scene in almost complete anonymity. The lack of a physical evidence trail at the scene of the crime makes detection and prosecution of computer crimes more difficult than ordinary white collar crime and reduces the risks for perpetrators of computer crime.

Today, the Internet is the medium of choice for electronic commerce. The Internet was not designed to be secure. Rather, the Internet was designed to support interoperability between heterogeneous computer platforms using a common protocol for communication. Providing a simple common set of protocols (TCP/IP) enabled maximum connectivity to the Internet without imposing undue burdens on each platform. In contrast, if the Internet protocol required each computer to support an encryption standard, a digital signature standard, or a key exchange standard, the Internet never would have gotten off the ground. The Internet protocol is a clear example of trading off security for flexibility. With the availability of the Internet to each desktop, the exposure of confidential or proprietary resources to the Internet is a threat to corporations. While encryption technology is very effective in providing privacy in Internet communications, it does little to close holes in the network through which 
intruders gain access to sensitive assets. Firewalls are the most effective strategy for preventing unauthorized access to network services. However, even firewalls are sensitive to data-driven attacks through legitimate network services [2]. These topics are discussed in more details in Section 1.2.

Currently, a plethora of encryption protocols exists for securing data transactions over the Internet, including Secure Sockets Layer (SSL), Secure HTTP (S-HTTP), Secure MIME (S/MIME), Secure Electronic Transaction (SET), CyberCash's Secure Internet Payment System, and DigiCash's e-cash. The vendors that support and sell implementations of these protocols would like consumers and businesses to think that e-commerce is secure when using these protocols. They are only partially correct. The truth is there is no silver bullet to e-commerce security. Securing the data transaction via encryption protocols provides privacy for data sent over the Internet. It does not protect a company's e-commerce server system from attack. It does not provide end users protection against malicious mobile code downloaded from rogue Web sites. Any e-commerce transaction is processed by a number of different components, any of which may be a weak link in the security of the transaction. The security of the system is only as strong as its weakest link. Computer criminals are unlikely to attempt to attack even weak encryption protocols (e.g., 48-bit encryption) when breaking into network servers is so much easier. Thus, the security of the components executing e-commerce transactions should be relatively uniform in strength. If one component is significantly stronger than others, then the weaker components are more likely to be attacked and the system compromised.

Recognizing that the security of the data transport in e-commerce systems is significantly stronger than other components in e-commerce systems, the weak links in e-commerce security are highlighted in the rest of this paper including client software such as Web browsers, server software including network services and the operating system, and CGI scripts.

\subsection{WEAK LINKS}

Electronic commerce systems are often implemented as a three-tiered architecture consisting of client software, network server software, and back-end databases. In addition, a middleware layer exists between network servers and the back-end databases that processes e-commerce transactions and updates the databases. Vulnerabilities in any of these software components can compromise the security of the entire enterprise.

The most serious risks posed to e-commerce security today are borne by the merchants themselves. Consumers' liability due to credit card fraud is often limited. For instance, if a consumer's credit card numbers are stolen and fraudulently used, the consumer's liability is restricted to U.S. $\$ 50$ by most credit lenders. On the other hand, the amount of credit card fraud that occurs on an annual basis totals in the billions of dollars and is accepted as the cost of business. In spite of the staggering losses due to credit card fraud, the banking industry is not complacent about fraud. Rather, the strategy used to address 
credit card fraud is risk management. Fraud risks are identified and costs for mitigating them calculated. When the return on investment is sufficient, legal enforcement is applied and preventative mechanisms are deployed. Risk management is equally effective in dealing with threats to computer security. No commercial system will ever be 100 percent secure to all possible threats. Rather, the benefit of preventing computer crimes from occurring must be weighed against the cost of protecting digital assets from attack.

The liability for merchants due to computer crimes is rising significantly each year. A joint study by the Computer Security Institute (CSI) and the U.S. Federal Bureau of Investigations (FBI) in 1998 found that the total financial losses reported by corporations over the previous year rose $36 \%$ from a similar study in 1997 to $\$ 136$ million [7]. Over $64 \%$ of respondents in the study reported computer security breaches within the past 12 months, representing a $16 \%$ increase in computer security violations over the previous year's findings. Finally, the most serious losses occurred through unauthorized access by insiders. This last point underscores the need for maintaining internal host security at the merchant site in spite of unknown threats from the Internet. Before discussing the weak links in the merchant side, the risks posed to end users are first described.

\subsection{WEB CLIENTS}

The vast majority of all vertical market (business to consumer) transactions are performed using Web browsers as the front-end. Web browsers today pose risks to end users' security and privacy. The greatest threat to end users' security and privacy is simply lack of knowledge about the risks of using Web browsers to visit untrusted sites. In addition, a large amount of privacy concerns can be alleviated by simply knowing what personal information is captured by Web sites when surfing the Web. Additional steps can be taken from preventing personal information from being released as described in this section. Similarly, hazards imposed by executable content can be addressed by disabling their execution from untrusted Web sites by configuring the browser appropriately.

Casting executable content aside for the moment, flaws in Web browsers themselves can cause security problems for end users. Employees who use browsers to "surf the Net" may potentially compromise the security of the corporate systems. The first issue companies must wrestle with is whether or not to trust the Web browser itself. Most browsers are given the privilege to execute programs locally, to write to user disks, to upload and download files and programs from the Internet. The consumer must trust that the browser software is not performing any malicious actions such as corporate espionage on a file system.

As an example of client browser vulnerabilities, consider how users of Microsoft's Internet Explorer (IE) version 3.0 can be tricked into executing any program on their machines at the behest of a remote server [5]. The discovery of this bug in the Internet Explorer has resulted in careful scrutiny of the Internet Explorer software, resulting in yet more security bug findings by groups 
at MIT and the University of Maryland [4]. The bug allows a Web page master to embed "shortcuts" from a Web page to a program anywhere on a user's host machine. A shortcut is a method for executing a program on a Win32 machine from the desktop. The security problem is that a computer user might hit a link on someone else's Web page (residing on a remote server) that causes the execution of a program that resides on the user's local machine. What this means is that someone else whom you don't necessarily know or trust can cause certain programs to execute on your desktop. What is particularly insidious about this bug is that it requires no executable content to be downloaded, such as ActiveX controls or Java applets, and the program can be executed in spite of the highest level of security set in the IE browser. The latter point is not surprising since the security levels can only prevent executable content from executing on the user's local machine, rather than preventing a shortcut to another program on the desktop.

The bug is a direct result of integrating the IE browser with the Windows desktop. While the ability to create a shortcut from a Web page to a user's desktop may have been viewed as a "feature" during the software design, it is in fact a bug that can be used against a user to violate the security of the user's machine. The bug allows a Web page writer to include ".URL" and ".LNK" files on a Web page. These files are shortcuts to executing programs that may exist on the user's Win32 machine. For this attack to work, the programs must exist on the desktop. There are, however, many programs that exist on a Windows desktop that can be used maliciously. For example, a shortcut link can be made to the Windows COMMAND.COM executable program to execute DOS commands that can modify the file system. With some degree of sophistication, the problem can be made much worse. By combining shortcut links with clientside executable scripts such as VBScript or JavaScript, malicious commands or programs can be downloaded into the IE cache as a batch file and then executed in sequence. This problem has since been corrected in subsequent release versions of the Internet Explorer.

\subsubsection{Privacy concerns}

Privacy issues in e-commerce are becoming highly visible in the media as well as in the U.S. Congress. Simply by surfing to different Web sites, consumers are giving out personal information about themselves. For instance, Web sites can and often do collect information about their Web site visitors including their name, email address, their Internet domain, machine name, platform type, and browser type. Some issuers of digital certificates, which are used to authenticate users to Web sites, shamelessly ask for and incorporate personal information such as age, gender, and profession in the digital certificates read by Web sites. This information, in turn, is used for directed marketing as well as profiling of visitors.

Cookies are another Web technology that can be used to intrude on end users' privacy. Cookies are data that are sent back and forth between the Web server and client to maintain state between Web connections. Cookies 
are stored on the user's own disk and retrieved the next time the user visits the same site. As such, Web sites can maintain persistent information about individual's browsing and shopping habits without knowledge or approval of the individual. The best known example of this strategy is by the company Doubleclick (ad.doubleclick.net). Doubleclick uses cookies to profile an individual's browsing habits. These browsing habits, in turn, are used for directed marketing to users who hit Web sites that use Doubleclick's services. Any time a user hits a site using Doubleclick, cookies are set to update the user's profile. For instance, if the user hits a search engine site and types the keywords "computer security", the user's individual profile will be updated to reflect that user's interest and on the page that is subsequently returned, banners of computer security vendors may be displayed. While directed marketing can be argued as benefiting both consumer and vendor, the databases built up on individual shopping/browsing habits can also be construed as an invasion of privacy. The safest way to prevent organizations to collect personal information is to browse through a proxy that removes the personal information. The anonymizer site (www.anonymizer.com) provides this service free of charge. Web proxies can be configured in house to do the same.

\subsubsection{Executable content}

Executable content poses privacy and security risks to end users, too. Java applets, ActiveX controls, Javascripts, and VBscripts are all examples of executable content. Others include PostScript files, multi-media files for browser plug-ins (e.g., .avi and .wav files), and mail attachments such as MS Word files. All of these forms of executable content are often downloaded or shared in e-commerce activities. Recently, the introduction of push technology into Web browsers has opened new vulnerabilities in the desktop by introducing scheduled content delivery including executable content. Executable content is simply another term for a computer program. Whenever computer programs are downloaded and executed on end users' machines, they execute with the privilege of the end user. Therefore, it is possible to program an ActiveX control that is placed on a Web page to download to a user's machine and read their mail files and send this information back to the Web server. The notorious Computer Chaos Club out of Germany demonstrated the ability to download a seemingly benign ActiveX control that in fact scheduled electronic transfers of funds from the user's account (set up by Quicken personal financial software) to a numbered Swiss bank account [1]. Similarly, it is possible to write JavaScripts to spy on all Web pages a user visits and send this information back to the Web site that sent the JavaScript.

1.3.2.1 Java applets. Java applets are mobile Java programs. That is, Java applets can be automatically downloaded from any Web page and run within the user's Web browser. Because the browser runs with the privilege of the user, the potential exists for Java applets to gain access to sensitive files on the user's desktop or to even execute commands with the user's full privileges. 
Java applets should not be confused with Java applications, which like any other full-featured program, have unrestricted access to system resources. Because Java applets automatically download and execute on the user's machine when its hosting Web site is hit, Java applets are considered untrusted code that must be carefully constrained. For this reason, the inventors of Java created a "sandbox" for Java applets in which Java applets may safely execute without posing risks to the user's security or privacy.

The Java sandbox poses a technological solution to constraining potentially malicious applet behavior. For instance, Java applets are not permitted to access the local file system. Also, Java applets are not allowed to make network connections except back to the originating site, nor can they listen to network connections made to the user's machine. The Java sandbox is enforced by three technologies: the bytecode verifier, the applet class loader, and the security manager [6]. The three technologies work in concert to prevent an applet from abusing its restricted privileges. Because each provides a different function, a flaw in any one can break the whole sandbox. For this reason, not only must their design be solid, but their implementations must be correct. The complexity of the functions that each technology provides makes correct implementations a difficult goal to attain in practice.

The Java security problems found to date have been a direct result of flaws in the implementations of the three components of the Java sandbox. Despite the efforts of JavaSoft in creating a sandbox, the Java security model has been broken on more than one occasion [6]. The Java security model depends on the enforcement of type safety in the language. Dynamic class loading in Java applets makes static type checking infeasible. Hence, the necessity for the three-pronged approach to the sandbox. Attack applets that are able to break type safety are effectively able to break out of the sandbox and completely compromise the system. Type safety flaws in the Java Virtual Machine (JVM) have largely been found by researchers in laboratories and since corrected by the vendor. Not surprisingly, flaws in the Java security model are usually found with each new release of the Java Developers Kit (JDK). With the release of JDK 1.2, a new security model for Java applets based on code signing is supported. This model effectively opens the sandbox to allow cryptographically signed applets to access system resources. If an applet has the correct signature to access the file system, for instance, it may be allowed to read or write files. Unsigned applets will still be restricted by the sandbox model. The problems the code signing model introduces are that every site must create, implement, and administer its own security policy for applets. Requiring sites to develop and administer their own security policies has proven to be impractical to date.

1.3.2.2 ActiveX controls. In contrast to the Java security model, ActiveX controls rely on a trust-based model for preventing malicious controls from executing. An ActiveX control is simply a program wrapped in a pre-specified interface that the Internet Explorer browser can execute. The program executes with the full rights and privileges of the browser. As such any ActiveX 
control can access any files on the user's machine, can delete, steal, or modify these files, and can execute commands on the user's machine. There are no constraints on the behavior of ActiveX controls. The ActiveX Exploder site (www.halcyon.com/mclain/ActiveX) illustrates this property well. The ActiveX control automatically downloaded, installed, and executed from this site will shut down a Windows machine.

The only technology imposed on ActiveX controls to prevent potentially malicious behavior is the control that requires user approval before installing the control. Prior to downloading and installing a new ActiveX control, a dialog box is popped up in the user interface. If the ActiveX control has a signed certificate, the certificate can be displayed to show which organization or individual is endorsing the control. If the user trusts the endorser, then the control will be downloaded and execution will begin. However, there is no technology to prevent a malicious control at this point to violate the security or privacy of the end user. As a result, the security model is totally trust-based. Users must make their own decisions on whether the control is trustworthy or not. Caution must be executed before agreeing to install and execute an ActiveX control.

1.3.2.3 Push technology. The final type of executable content to be considered here is known as push technology. Push technology turns the Web paradigm on its head. Web surfers are used to finding a Web site and requesting information. The information is pulled into the user's browser. With push technology, users still have to determine which Web sites they want information from, but once selected, the Web sites take matters into their own hands and push information to the browser without the user's prodding. Web sites who push active content are similar to their counterparts in the TV and radio industry. Essentially, these sites broadcast their content. Users need only "tune" their browsers to their channel. Hence, the concept of "active channels", now being pushed by Microsoft in the Internet Explorer 4.0. The idea is to get the latest updates on information without having to request it, since presumably you will not know when to request updated information.

The first well-known adopters of push technology came in the form of PointCast and Marimba. PointCast Network is a program that exploits push technology to distribute news over the Internet. PointCast broadcasts news, stock updates, sports scores, weather, and other dynamic content on a seemingly continuous basis.

Unlike the prevalent pull paradigm of the Web, push technology works on the principle of passive acceptance of data. That is, the client always accepts data pushed from the content provider, without control over what data is being sent. In the pull model, a client actively requests data from a Web site. Push technology, on the other hand, requires this decision to be made once. That is, the user subscribes to a channel (Web site) once and from that point on any and all content that matches your personal filter is downloaded. Bear in mind, the customizations are not geared around filtering out viruses. This 
gives the subscribed sites a great deal of leverage to send any data of their choosing. For example, a Web site can send not only updates of news, but also active content, digital images, plug-ins, and even software patches to update the network client on-the-fly. Since the client often belongs to one of the subscribers (e.g., PointCast and Microsoft), the client can be programmed to serve any number of functions. The client can be an interpreter to execute commands sent from broadcasters. For the more paranoid of mind, the network client can be used to spy on user's networked drives and send this data back over a network socket. How difficult would such an attack be? Remember the network client will have full system privileges as any other program running on your desktop. Also remember client approval is granted a priori via the subscription for downloading content over an active channel. Consider that the client (e.g., the IE 4.0) can download, install and execute executable content such as ActiveX controls at any point in the future. This means that it is possible to write an ActiveX control, or even a trusted Java applet to download to targeted clients (subscribers) and perform nefarious functions such as spying on their hard drives or even deleting files. Is this a stretch of the imagination? Perhaps. But the mechanism will be technologically built in to your desktop machine.

Other security concerns over push technology center around the updates of software. Network clients that support push technology can immediately update themselves with each new patch or each new release version of the software. This technique by itself can go a long way towards making networked machines more secure. Every time a software flaw is found in the network client, the network client can reach back to the vendor, download the patch, install it, and fortify itself against known attacks. One downside of the technique is the fact that the network client is downloading executables that can alter its functionality. The question is how safe are these executables? Is it possible that they could be downloaded from a rogue organization posing as the vendor? The answer is yes. Domain name spoofing is a well-known Internet attack. The attack works by fooling a DNS server to resolve a network address to an incorrect IP address belonging to the perpetrator. The perpetrator could then download its own version of the software modified to perform its objectives, such as spying on your hard drive. Can this attack be prevented? Yes. Using digital signatures, all executables can be signed to provide proof positive of the identity of the software publisher and to determine if the software has been corrupted in transit. This system is not perfect, however. The system is based on trust. You must trust each of your content providers to not download any malicious content. Even with digital signatures, a "trusted" organization can still exploit the push technology for its own gain at the expense of selected targets. Since downloading of content occurs at scheduled intervals, rather than at the behest of the end user, this malicious content can be downloaded and executed while the user is asleep at night or on a coffee break. This leaves the end user unaware of what happened and the content can erase all traces of any nefarious activity since it is given full access to the system. 
Are these reasons enough to not use push technology? Not unless you are using it on an enterprise-critical or mission-critical machine where the compromise of your digital assets could result in severe consequences. It is important to note that at the time of this writing, no attacks through push technology are known. The most important step users can take is to educate themselves of the risks and manage them appropriately.

\subsection{NETWORK SERVERS}

Clearly, a host of security and privacy issues are raised by the Web browsers that everyone now uses. Education is the best antidote to the risks of executable content to users. As discussed earlier, secure data transaction protocols can provide strong privacy for data transported in on-line sessions. The weak links in e-commerce security are on both ends of the network connection. Gene Spafford, a computer security researcher at Purdue University, made an interesting comment on the disparity between data transaction security and the security of the client and network server software:

Using encryption on the Internet is the equivalent of arranging an armored car to deliver credit-card information from someone living in a cardboard box to someone living on a park bench.

In the analogy of on-line commerce, users live in an environment as secure as a park bench, while the network servers are as secure as a cardboard box in the physical world. Clearly, if someone really wanted to steal a credit card number, it would be foolish to attack the armored car rather than either the cardboard box or the park bench.

Network server security is one of the most important components to secure of all e-commerce system components. The reason is that network servers are the gateway from the untrusted Internet to a company's proprietary digital assets. As such, they must be guarded against the types of threats posed by malicious computer hackers.

The most widely used technology for protecting network servers and internal digital assets is the firewall. Firewalls are the first line of defense against external attacks. A firewall is placed between the computer network to be protected and the network that is considered to be a security threat. Though firewalls are typically used to isolate local area networks within a company from the Internet, firewalls are also used to partition, isolate, and control access between internal corporate networks. Firewalls are usually a combination of filtering routers and application proxies that run on a dedicated machine.

Firewalls provide control over which network services are offered to the Internet or the external network at-large. An easy way to secure a network from external threats is simply to disconnect all access to and from the Internet. Since some Internet services such as mail, Web access, and FTP are essential in today's corporations to do business, disconnecting from the Internet is not a feasible nor a strategic option. On the other hand, simply connecting all internal machines to the Internet without forethought to computer security can place corporate assets at risk. Firewalls are a compromise solution between 
these two extreme positions of security and insecurity. Even though rigorous access controls can be imposed on the types of network services offered, there is the potential for attacks to be waged against a corporate network through errors in configuration of the firewall, around the firewall through backdoors, or even through legitimate requests over network services offered through the firewall.

While firewalls are useful for thwarting attacks launched through unintended network services, there is little that firewalls can do to prevent data-driven attacks through legitimate requests made to offered services. These types of attacks use the legitimate grammar of the protocol and creative license to trick software on the inside of the firewall to act on behalf of a remote user in violation of the security policy of the site.

Firewalls are ineffective at thwarting data-driven types of attacks through legitimate network service requests. One class of attacks exploits weaknesses in network applications running on a server. For example, sendmail is one of the most commonly used mail servers used on Unix machines. Throughout its long history (sendmail is now on version 8 approaching version 9) sendmail has been rife with errors that have resulted in security vulnerabilities. For example, in the past when sendmail was compiled in "debug" mode, it allowed untrusted outside users unrestricted access to the system . Even now, securityrelated bugs in sendmail are usually discovered with each subsequent release version. The problem is not that sendmail is poorly written, rather, the size and complexity of the sendmail program make a bug-free implementation a near impossibility.

Firewalls can do little to prevent program errors in an application server from being exploited through legitimate requests to the server. They can, however, limit the extent of the damage. A firewall proxy can create an artificially small file system around an executing application server. By creating this "jail cell" around a server, if the server program is compromised by an outside request, then the extent of damage that can be caused by the intrusion is limited to the scope of the jail cell. In the case of sendmail, a data-driven attack that is able to obtain shell access on the server through a bug in sendmail will only be able to access files and/or programs in the file system that is defined by the the jail cell ${ }^{1}$. Of course, any mail that is within the scope of the jail cell may be vulnerable to eavesdropping by a subverted sendmail program. The key to addressing the firewall's vulnerability to data-driven attacks is to stay on top of the latest holes found in server-side software and to patch the software as fixes are released ${ }^{2}$.

Network servers are vulnerable to external threats due to errors in configuration, flaws in the server software and interface scripts, inappropriate access controls to the back-end databases, and security holes in the operating system

\footnotetext{
1The Unix chroot command is used to define the "jail cell".

${ }^{2}$ Bugtraq (www.netspace.org/lsv-archive/bugtraq.html) is the premier forum for reporting security-related software bugs.
} 
that underlies the network server. The setup and configuration of a network server can be complex and, similar to firewalls, simple errors in configuring the network server may have drastic security implications. Most network servers consist of network services such as a Web server, a mail server, and sometimes other network services such as file transfer protocol (FTP), and news (NNTP). Configuring these services securely is a formidable task even for experienced administrators. Most of the problems in security of corporate systems are a direct result of errors in configuration. The rest are flaws in the actual software source code. Configuration errors can lead to privilege escalation where an unauthorized and untrusted user gains a level of privilege for unauthorized access to corporate information systems. As an example, consider a system administrator who installs and configures a Web server. The system administrator knows that the server must start up as the super user - the user account with highest privileges - in order to listen to port 80, the standard port for Web requests. Without realizing the security implications, the system administrator also sets the executing privilege of the Web server to the super user. Now, any actions the server takes will have the force of the super user. This means that if an attacker is able to subvert the server or any of the programs the server calls, the attacker will now have the privilege to read, modify, delete, or create any file on the system.

Software is mostly configured to meet the functional requirements of the organization, e.g., providing access to corporate intranets from remote logins, rather than configured to meet requirements of corporate security policy. Most network software that is installed out-of-the-box is configured by default to provide maximum functionality, rather than security. Unless configured to meet a company's own security policy, the network services will probably be vulnerable to attack. The default configurations of network servers are known as the deadly defaults. Therefore, the firewall, the network servers, the middleware, and access to the back-end databases must all be configured to uphold each site's own security policy. In [3], common errors in configuration of Web servers that are exploited for security breaches are described.

\subsection{SERVER-SIDE MIDDLEWARE}

Aside from the network server, perhaps a more dangerous form of software that has emerged in on-line applications is the Common Gateway Interface (CGI) script. CGI scripts and other middleware are server-side programs that execute when called by the Web server in response to a Web request. Simple CGI scripts may increment a counter each time a Web page is accessed. Others may support customer feedback via mail. More sophisticated CGI scripts perform online transaction processing tasks required of on-line commercial transactions. For example, a CGI script may submit a customer query to an on-line database to find out the customer's investment portfolio balance.

Because CGI scripts execute in response to a remote user's request and typically process user input directly, the danger exists for a user to be able to manipulate the CGI script into giving system privileges to the untrusted 
user. This is particularly true for electronic commerce, where in order for any transaction to occur, user input is necessary, an application must be executed, and files must be updated. It is the sheer power of CGI to execute interesting applications that makes it so dangerous to corporate security.

CGI scripts are written in interpreted languages such as Perl and Python or in compiled languages such as C. Perl is popular for CGI scripts because of the ability to rapidly construct applications and the ability to parse text from user input easily. However, languages such as Perl also provide a great deal of power for executing system commands that can be exploited by malicious users. For example, certain Perl commands such as eval(), system(), backquotes ('), pipes, and exec() can potentially result in system commands being executed on the network server host at the discretion of an unknown and untrusted remote user. Using these commands in CGI scripts is especially dangerous because unexpected malicious user input from remote systems can easily turn commonly used Perl commands into vehicles for intrusions.

Several steps can be taken to mitigate the dangers of CGI scripts. First, users should not be allowed to place their own CGI scripts on the Web server. Users are much less likely to test and verify that their scripts do not pose a security hazard, especially if they do not have the technology to perform security analysis. System administrators must be aware of stray CGI scripts that get placed on the server. These scripts can often be a backdoor that hackers (or potentially malicious internal users) leave behind to allow unauthorized entry into a system. The Web server should be configured such that CGI programs can only be executed from a single directory (with appropriate access control). If configured successfully, this measure can reduce the threat of users creating CGI scripts in their home directories. Even CGI scripts that are distributed with Web servers, downloaded from the Internet, or purchased commercially, should be viewed with suspicion. More to the point, all CGI scripts should be tested rigorously for security holes.

Scripting or interpreted languages such as Perl should be avoided. While compiled languages such as $\mathrm{C}$ can be equally hazardous, the scripting languages make it easier for users to unintentionally code dangerous constructs. Even if the system administrator decides that a CGI script is safe, it is wise to keep the source code for the CGI scripts hidden from the outside world. If a person outside the organization can download the source, then the source can be analyzed for vulnerabilities and potentially exploited later. Finally, every CGI program on the server must be accounted for in terms of its purpose, origin, and modifications. If the program does not serve a business function of the Web server it should be removed. This will eliminate most of the demo CGI scripts that are distributed with the Web server software. Once a stable set of CGI programs is established, a digital hash of the program (using MD5, for example) executables should be made. This will allow any modifications of the programs to be detected in the future by comparing subsequent hashes with the original digital hash. 


\subsection{CONCLUSIONS}

Electronic commerce systems are vulnerable to malicious attack through many different software components. This paper describes vulnerabilities in Web clients, firewalls, network servers, server-side middleware, and databases that can be exploited in compromising e-commerce security. In current e-commerce systems, the lion's share of security work has been focused on data transaction protocols. While these protocol implementations are not free from errors by any means, they represent the most secure component in e-commerce systems today. As a result, a disproportionate amount of attacks against e-commerce systems have been focused against server-side systems and users' client software.

\section{Acknowledgments}

This work has been supported by the Advanced Technology Program of NIST under Cooperative Agreement \# 70NANB7H3049.

\section{References}

[1] Klaus Brunnstein. Hostile activex control demonstrated. RISKS Digest, 18(82), February 1997.

[2] A.K. Ghosh. Securing electronic commerce: Moving beyond cryptography. EDI Forum Journal of Electronic Commerce, 10(4):77-85, 1997.

[3] A.K. Ghosh. E-Commerce Security: Weak Links, Best Defenses. John Wiley \& Sons, New York, NY, 1998. ISBN 0-471-19223-6.

[4] C. Haney. That was the buggy week that was. Available online: www.techwire.com, March 71997.

[5] Cybersnot Industries. Internet explorer bug (version 2.0 (4.70.1155)). Available online: www.cybersnot.com, February 271997.

[6] G. McGraw and E. Felten. Java Security: Hostile Applets, Holes, and Antidotes. John Wiley and Sons, New York, 1996.

[7] Press release. Annual cost of computer crime rise alarmingly. 1998 CSI/FBI Computer Crime and Security Survey. Available online:www.gocsi.com, March 41998.

[8] G.W. Treese. Internet security: To worry or not to worry? EDI Forum Journal of Electronic Commerce, 10(4):54-58, 1997. 